\title{
BILINEAR DIFFERENTIAL OPERATORS: PROJECTIVELY EQUIVARIANT SYMBOL AND QUANTIZATION MAPS
}

\author{
TAHER Bichr, JAMEl Boujelben AND KHALED TOUNSI
}

(Received March 10, 2014, revised July 3, 2014)

\begin{abstract}
We study the space of bilinear differential operators on weighted densities as a module over $\operatorname{sl}(2, \mathbb{R})$. We introduce the corresponding space of symbols and we prove the existence and the uniqueness of canonical projective equivariant symbol and quantization maps.
\end{abstract}

1. Introduction. The general problem of quantization is to associate linear operators on a Hilbert space with Hamiltonian functions on a symplectic manifold. In the simplest situation when the symplectic manifold is a cotangent bundle $T^{*} M$ over a manifold $M$, the usual quantization procedure consists of establishing a correspondence between the space $\operatorname{Pol}\left(T^{*} M\right)$ and the space $\mathcal{D}(M)$ of linear differential operators on $M$ called a symbol map:

$$
\sigma: \mathcal{D}(M) \rightarrow \operatorname{Pol}\left(T^{*} M\right) .
$$

The inverse $\mathcal{Q}=\sigma^{-1}: \operatorname{Pol}\left(T^{*} M\right) \rightarrow \mathcal{D}(M)$ is called the quatization map. Generally, there is no quantization map (and symbol map) equivariant with respect to the action the group $\operatorname{Diff}(M)$ of diffeomorphisms of $M$ and the Lie algebra $\operatorname{Vect}(M)$ of vector fields on $M$. Two spaces: $\operatorname{Pol}\left(T^{*} M\right)$ and $\mathcal{D}(M)$ are not isomorphic as modules over $\operatorname{Diff}(M) \operatorname{or} \operatorname{Vect}(M)$.

Let $M$ be a smooth manifold. We consider the space $\mathcal{F}_{\lambda}(M)$ of tensor densities of degree $\lambda$ on $M$ (i.e., the space of sections of the line bundle $\Delta_{\lambda}(M)=\left|\Lambda^{n} T^{*} M\right|^{\otimes \lambda}$ over $M$ ). Clearly, $\mathcal{F}_{0}(M) \cong C^{\infty}(M)$ as a $\operatorname{Vect}(M)$-module. Denote $\mathcal{D}_{\lambda, \mu}(M)$ the space of linear differential operators from $\mathcal{F}_{\lambda}(M)$ to $\mathcal{F}_{\mu}(M)$. This space is an associative (and, therefore, a Lie) algebra with the filtration by the order of differentiation:

$$
\mathcal{D}_{\lambda, \mu}^{0}(M) \subset \mathcal{D}_{\lambda, \mu}^{1}(M) \cdots \subset \mathcal{D}_{\lambda, \mu}^{k}(M) \subset \cdots .
$$

On the other hand, we consider the space $\mathcal{S}(M)$ of symmetric contravariant tensor fields on $M$ (i.e., $\mathcal{S}(M)=\Gamma(S T M)$ ). As a $\operatorname{Vect}(M)$-module it is isomorphic to the space $\operatorname{Pol}\left(T^{*} M\right)$. Therefore, $\mathcal{S}(M)$ is a Poisson algebra with a natural graduation given by the decomposition

$$
\mathcal{S}(M)=\bigoplus_{k=0}^{\infty} \mathcal{S}^{k}(M)
$$

2010 Mathematics Subject Classification. Primary 53D55.

Key words and phrases. Bilinear differential operators, densities, projective algebra, symbol map. 
where $\mathcal{S}^{k}(M)$ is the space of $k$-th order tensor fields. The algebra $\mathcal{S}(M)$ is naturally identified with the associated graded algebra $\operatorname{gr}\left(\mathcal{D}_{\lambda, \mu}(M)\right)$, that is

$$
\mathcal{D}_{\lambda, \mu}^{k}(M) / \mathcal{D}_{\lambda, \mu}^{k-1}(M) \cong \mathcal{S}^{k}(M) .
$$

The corresponding projection $\sigma_{k}: D_{\lambda, \mu}^{k}(M) \rightarrow \mathcal{S}^{k}(M)$ is called the (principal) symbol.

Several works have been interested in the study of the problem of existence and uniqueness of symbol and quantization maps. In [2], It was proven the existence and the uniqueness of a conformally equivariant symbol calculus and quantization on any conformally flat pseudoRiemannian manifold. For the flat manifold $M=\mathbb{R}^{n}$, the authors define in [6] the unique (up to normalization) symbol map from the 1 -parameter space $\mathcal{D}_{\lambda}\left(\mathbb{R}^{n}\right)$ (i.e., $\lambda=\mu$ ) of linear differential operators on $\mathcal{F}_{\lambda}\left(\mathbb{R}^{n}\right)$ to the space of polynomial on fibers functions on $T^{*} \mathbb{R}^{n}$, equivariant with respect to the Lie algebra of projective transformations $\operatorname{sl}(n+1, \mathbb{R}) \subset \operatorname{Vect}\left(\mathbb{R}^{n}\right)$. This implies that $\mathcal{D}_{\lambda}\left(\mathbb{R}^{n}\right)$ is isomorphic to $\operatorname{Pol}\left(T^{*} \mathbb{R}^{n}\right)$ as a module over $\operatorname{sl}(n+1, \mathbb{R})$. The same result holds for manifolds endowed with a projective structure (an atlas with linear-fractional coordinate transformations). In the one-dimensional case $(n=1)$, in [3] and [4], thanks to the $\operatorname{sl}(2, \mathbb{R})$-equivariant symbol map, a complete description of the modules $\mathcal{D}_{\lambda, \mu}^{k}(\mathbb{R})$ was given. In $[1], \operatorname{sl}(2, \mathbb{R})$-equivariant symbol (and quantization) map was obtained in a more general situation of pseudodifferential operators. In the case of the supermanifold $S^{1 \mid 1}$ equipped with the standard contact structure, the authors study in [5] the space $\mathcal{D}_{\lambda, \mu}\left(S^{1 \mid 1}\right)$ as a module over the Möbius superalgebra osp(1|2). They introduce the canonical isomorphism between this space and the corresponding space of symbols and find all cases where such an isomorphism does not exist.

The main object of our study is the space of bilinear differential operators acting on weighted densities on $\mathbb{R}$, the space $\mathcal{D}_{\lambda_{1}, \lambda_{2}, \mu}$ of bilinear differential operators from $\mathcal{F}_{\lambda_{1}} \otimes \mathcal{F}_{\lambda_{2}}$ to $\mathcal{F}_{\mu}$ is also a $\operatorname{Vect}(\mathbb{R})$ (and a $\operatorname{Diff}(\mathbb{R})$ )-module. Each module $\mathcal{D}_{\lambda_{1}, \lambda_{2}, \mu}$ has a natural filtration by the order of differential operators. The graded module $\operatorname{gr}\left(\mathcal{D}_{\lambda_{1}, \lambda_{2}, \mu}\right)$, also called the space of symbols and denoted by $\mathcal{S}_{\lambda_{1}, \lambda_{2}, \mu}$, depends only on the shift, $\delta=\mu-\lambda_{1}-\lambda_{2}$, of the weights. Moreover, we show that, as a $\operatorname{Vect}(\mathbb{R})$-module, $\mathcal{S}_{\lambda_{1}, \lambda_{2}, \mu}$ is decomposed as

$$
\bigoplus_{k=0}^{\infty} \bigoplus_{\ell=0}^{k} \bigoplus_{i+j=\ell} \mathcal{F}_{\frac{\delta}{2}+i} \otimes \mathcal{F}_{\frac{\delta}{2}+j} .
$$

We restrict the $\operatorname{Vect}(\mathbb{R})$-module structures to the particular subalgebra $\operatorname{sl}(2, \mathbb{R})$ and look for $\mathrm{sl}(2, \mathbb{R})$-isomorphisms called respectively projectively equivariant symbol and quantization maps:

$$
\sigma_{\lambda_{1}, \lambda_{2}, \mu}: \mathcal{D}_{\lambda_{1}, \lambda_{2}, \mu} \rightarrow \mathcal{S}_{\lambda_{1}, \lambda_{2}, \mu} ; \mathcal{Q}_{\lambda_{1}, \lambda_{2}, \mu}=\sigma_{\lambda_{1}, \lambda_{2}, \mu}^{-1}: \mathcal{S}_{\lambda_{1}, \lambda_{2}, \mu} \rightarrow \mathcal{D}_{\lambda_{1}, \lambda_{2}, \mu} .
$$

As the linear case, for almost all values $\left(\lambda_{1}, \lambda_{2}, \mu\right)$, we prove the existence and the uniqueness (up to normalization) of the $\operatorname{sl}(2, \mathbb{R}$ )-equivariant symbol map and calculate its explicit formula. We also calculate the quantization map $\mathcal{Q}=\sigma^{-1}$. The proof of the uniqness of the equivariant symbol map allows us to prove, that in terms of the $\mathrm{sl}(2, \mathbb{R})$-equivariant symbol, every isomorphisms of modules of bilinear differential operators has a 'diagonal' form. 
Finally, as an example, the case of modules of second order bilinear differential operators is investigated, we determine, for almost all values $\left(\lambda_{1}, \lambda_{2}, \mu\right)$, the isomorphisms of the modules $\mathcal{D}_{\lambda_{1}, \lambda_{2}, \mu}^{2}$.

We hope to be able, in the immediate future, using our results, to give a full description of the modules $\mathcal{D}_{\lambda_{1}, \lambda_{2}, \mu}^{k}$ for all integer $k$. A work in this framework is currently being.

2. Preliminaries. Denote by $\mathcal{F}_{\lambda}, \lambda \in \mathbb{R}$ (or $\mathbb{C}$ ), the space of densities of degree $-\lambda$ on $\mathbb{R}\left(\right.$ or $\left.S^{1}\right)$

$$
\Phi=\varphi(x)(d x)^{-\lambda} ; \varphi \in C^{\infty}(\mathbb{R}) .
$$

As a vector space, $\mathcal{F}_{\lambda}$ is isomorphic to $C^{\infty}(\mathbb{R})$. The Lie algebra Vect $(\mathbb{R})$ of vector fields and the group $\operatorname{Diff}(\mathbb{R})$ of all diffeomorphisms of $\mathbb{R}$ naturally act respectively on the space $\mathcal{F}_{\lambda}$ by

$$
L_{X}^{\lambda}(\Phi)=\left(X(x) \varphi^{\prime}(x)-\lambda X^{\prime}(x) \varphi(x)\right)(d x)^{-\lambda}:=L_{X}^{\lambda}(\varphi)(d x)^{-\lambda}
$$

where $X=X(x) \frac{d}{d x} \in \operatorname{Vect}(\mathbb{R})$ and

$$
\rho_{f^{-1}}^{\lambda}(\Phi)=\left(\left(f^{\prime}\right)^{-\lambda} . \varphi \circ f(x)\right)(d x)^{-\lambda} ; f \in \operatorname{Diff}(\mathbb{R}) .
$$

Obviously, $\forall \lambda_{1}, \lambda_{2} \in \mathbb{R}, \mathcal{F}_{\lambda_{1}} \times \mathcal{F}_{\lambda_{2}}$ is also a $\operatorname{Vect}(\mathbb{R})($ and a $\operatorname{Diff}(\mathbb{R})$ )-module with the action

$$
L_{X}^{\lambda_{1}, \lambda_{2}}\left(\Phi_{1}, \Phi_{2}\right)=\left(L_{X}^{\lambda_{1}}\left(\Phi_{1}\right), \Phi_{2}\right)+\left(\Phi_{1}, L_{X}^{\lambda_{2}}\left(\Phi_{2}\right)\right) .
$$

Consider the space of bilinear differential operators

$$
A: \mathcal{F}_{\lambda_{1}} \times \mathcal{F}_{\lambda_{2}} \rightarrow \mathcal{F}_{\mu}
$$

with arbitrary $\lambda_{1}, \lambda_{2}, \mu \in \mathbb{R}$. This space will be denoted by $\mathcal{D}_{\lambda_{1}, \lambda_{2}, \mu}$. The subspace of bilinear differential operators of order $\leq k$ will be denoted by $\mathcal{D}_{\lambda_{1}, \lambda_{2}, \mu}^{k}$. A bilinear differential operator $A \in \mathcal{D}_{\lambda_{1}, \lambda_{2}, \mu}^{k}$ is of the form

$$
A=\sum_{\ell=0}^{k} \sum_{i+j=\ell} a_{i, j}(x) \frac{d^{i}}{d x^{i}} \otimes \frac{d^{j}}{d x^{j}}
$$

where $a_{i, j}(x)$ are smooth functions. More precisely,

$$
A\left(\Phi_{1}, \Phi_{2}\right)=\left(\sum_{\ell=0}^{k} \sum_{i+j=\ell} a_{i, j}(x) \frac{d^{i} \varphi_{1}}{d x^{i}} \frac{d^{j} \varphi_{2}}{d x^{j}}\right)(d x)^{-\mu}
$$

where $\Phi_{1}=\varphi_{1}(x)(d x)^{-\lambda_{1}} \in \mathcal{F}_{\lambda_{1}}$ and $\Phi_{2}=\varphi_{2}(x)(d x)^{-\lambda_{2}} \in \mathcal{F}_{\lambda_{2}}$.

The Lie algebra $\operatorname{Vect}(\mathbb{R})$ acts on the space of bilinear differential operators as follows

$$
L_{X}^{\lambda_{1}, \lambda_{2}, \mu}(A)\left(\Phi_{1}, \Phi_{2}\right)=L_{X}^{\mu} \circ A\left(\Phi_{1}, \Phi_{2}\right)-A\left(L_{X}^{\lambda_{1}}\left(\Phi_{1}\right), \Phi_{2}\right)-A\left(\Phi_{1}, L_{X}^{\lambda_{2}}\left(\Phi_{2}\right)\right)
$$

where $L_{X}^{\lambda}$ is the action given in (2). For short we write

$$
L_{X}^{\lambda_{1}, \lambda_{2}, \mu}(A)=L_{X}^{\mu} \circ A-A \circ L_{X}^{\lambda_{1}, \lambda_{2}} .
$$

The space $\mathcal{D}_{\lambda_{1}, \lambda_{2}, \mu}$ is thus endowed, thanks to (8), with the $\operatorname{Vect}(\mathbb{R})$-module structure. In a similar manner, one can make the space $\mathcal{D}_{\lambda_{1}, \lambda_{2}, \mu}$ a $\operatorname{Diff}(\mathbb{R})$-module. 
The projective Lie algebra, i.e., the Lie subalgebra of the Lie algebra Vect $(\mathbb{R})$ of vector fields on $\mathbb{R}$ generated by the following vector fields:

$$
\frac{d}{d x}, x \frac{d}{d x}, x^{2} \frac{d}{d x}
$$

is isomorphic to $\operatorname{sl}(2, \mathbb{R})$. Theese vector fields are infinitesimal generators of (locally defined on $\mathbb{R}$ ) linear-fractional transformations. Note that the Lie subalgebra $\operatorname{sl}(2, \mathbb{R}) \subset \operatorname{Vect}(\mathbb{R})$ is maximal in the following sense. Given an arbitrary polynomial vector field $X \notin \operatorname{sl}(2, \mathbb{R})$, then, a Lie algebra generated by $X$ together with the vector fields, coincides with the whole Lie algebra of polynomial vector fields on $\mathbb{R}$. Obviously, $\mathcal{F}_{\lambda}$ and $\mathcal{D}_{\lambda_{1}, \lambda_{2}, \mu}$ are modules over the projective algebra $\mathrm{sl}(2, \mathbb{R})$.

\section{Modules of bilinear differential operators.}

3.1. The invariant $\delta=\mu-\lambda_{1}-\lambda_{2}$.

LEMma 3.1. For the Vect $(\mathbb{R})$-module $\mathcal{D}_{\lambda_{1}, \lambda_{2}, \mu}^{k}$, the difference $\delta=\mu-\lambda_{1}-\lambda_{2}$ of weights is an invariant. That is:

$$
\mathcal{D}_{\lambda_{1}, \lambda_{2}, \mu}^{k} \cong \mathcal{D}_{\rho_{1}, \rho_{2}, v}^{k} \Rightarrow \mu-\lambda_{1}-\lambda_{2}=v-\rho_{1}-\rho_{2} .
$$

PROOF. This is an immediate consequence of the equivariance with respect to the vector field $x \frac{d}{d x}$.

3.2. Adjoint modules. In this section, the following results and proofs are adapted from the unary case (see [3] and [4] for more details).

THEOREM 3.2. For each value of $k$, there exist the following isomorphisms of Vect $(\mathbb{R})$ modules:

$$
\mathcal{D}_{\lambda_{1}, \lambda_{2}, \mu}^{k} \cong \mathcal{D}_{-1-\mu, \lambda_{2},-1-\lambda_{1}}^{k} \cong \mathcal{D}_{\lambda_{1},-1-\mu,-1-\lambda_{2}}^{k} \cong \mathcal{D}_{-1-\mu, \lambda_{1},-1-\lambda_{2}}^{k} \cong \mathcal{D}_{\lambda_{2},-1-\mu,-1-\lambda_{1}}^{k} \cong \mathcal{D}_{\lambda_{2}, \lambda_{1}, \mu}^{k}
$$

given respectively by the $\mathrm{sl}(2)$-invariant maps

$$
\begin{aligned}
& C_{1}: A=\sum_{\ell=0}^{k} \sum_{i+j=\ell} a_{i, j} \frac{d^{i}}{d x^{i}} \otimes \frac{d^{j}}{d x^{j}} \mapsto C_{1}(A)=\sum_{\ell=0}^{k} \sum_{i+j=\ell}(-1)^{(i)} \sum_{s=0}^{i}\left(\begin{array}{l}
i \\
s
\end{array}\right) \frac{d^{i-s}}{d x^{i-s}} \circ a_{i, j} \otimes \frac{d^{j+s}}{d x^{j+s}} \\
& C_{2}: A=\sum_{\ell=0}^{k} \sum_{i+j=\ell} a_{i, j} \frac{d^{i}}{d x^{i}} \otimes \frac{d^{j}}{d x^{j}} \mapsto C_{2}(A)=\sum_{\ell=0}^{k} \sum_{i+j=\ell}(-1)^{(j)} \sum_{s=0}^{j}\left(\begin{array}{l}
j \\
s
\end{array}\right) \frac{d^{i+s}}{d x^{i+s}} \otimes \frac{d^{j-s}}{d x^{j-s}} \circ a_{i, j} \\
& C_{1} \circ C_{2}, C_{2} \circ C_{1} \text { and } C_{2} \circ C_{1} \circ C_{2}\left(\text { or } C_{1} \circ C_{2} \circ C_{1}\right) .
\end{aligned}
$$

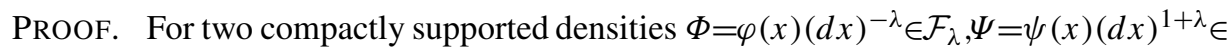
$\mathcal{F}_{-1-\lambda}$, we consider the $\operatorname{Vect}(\mathbb{R})$-invariant pairing

$$
\langle\Phi, \Psi\rangle=\int_{\mathbb{R}} \varphi(x) \psi(x) d x .
$$


Then, for $A \in \mathcal{D}_{\lambda_{1}, \lambda_{2}, \mu}^{k}$, there exists a unique bilinear differential operator $A_{1}^{*} \in \mathcal{D}_{-1-\mu, \lambda_{2},-1-\lambda_{1}}^{k}$ such that

$$
\left\langle A\left(\Phi_{1}, \Phi_{2}\right), \Psi\right\rangle=\left\langle\Phi_{1}, A_{1}^{*}\left(\Psi, \Phi_{2}\right)\right\rangle
$$

where $\Phi_{1} \in \mathcal{F}_{\lambda_{1}}, \Phi_{2} \in \mathcal{F}_{\lambda_{2}}$ and $\Psi \in \mathcal{F}_{-1-\mu}$. We easily show that the map $C_{1}: A \mapsto A_{1}^{*}$ is $\operatorname{Vect}(\mathbb{R})$-invariant, that is

$$
C_{1} \circ L_{X}^{\lambda_{1}, \lambda_{2}, \mu}=L_{X}^{-1-\mu, \lambda_{2},-1-\lambda_{1}} \circ C_{1} .
$$

The explicit expression of the map $C_{1}$ (or $C_{2}$ ) given in (13) is produced immediately trough integration by parts. Indeed, let $A=a_{i, j} \frac{d^{i}}{d x^{i}} \otimes \frac{d^{j}}{d x^{j}}, \Phi_{1}=\varphi_{1}(x)(d x)^{-\lambda_{1}} \in \mathcal{F}_{\lambda_{1}}, \Phi_{2}=$ $\varphi_{2}(x)(d x)^{-\lambda_{2}} \in \mathcal{F}_{\lambda_{2}}$ and $\Psi=\psi(x)(d x)^{1+\mu} \in \mathcal{F}_{-1-\mu}$. Then

$$
\begin{aligned}
\left\langle A\left(\Phi_{1}, \Phi_{2}\right), \Psi\right\rangle & =\int_{\mathbb{R}} a_{i, j}(x) \varphi_{1}(x)^{(i)} \varphi_{2}^{(j)}(x) \psi(x) d x \\
& =(-1) \int_{\mathbb{R}} \varphi_{1}(x)^{(i-1)}\left(a_{i, j}(x) \varphi_{2}^{(j)}(x) \psi(x)\right)^{\prime} d x \\
& \vdots \\
& =(-1)^{(i)} \int_{\mathbb{R}} \varphi_{1}(x)\left(a_{i, j}(x) \varphi_{2}^{(j)}(x) \psi(x)\right)^{(i)} d x \\
& =(-1)^{(i)} \int_{\mathbb{R}} \varphi_{1}(x) \sum_{s=0}^{i}\left(\begin{array}{l}
i \\
s
\end{array}\right)\left(\psi(x) a_{i, j}(x)\right)^{(i-s)} \varphi_{2}^{(j+s)}(x) d x \\
& =\left\langle\Phi_{1}, A_{1}^{*}\left(\Psi, \Phi_{2}\right)\right\rangle
\end{aligned}
$$

where $A_{1}^{*}=(-1)^{(i)} \sum_{s=0}^{i}\left(\begin{array}{l}i \\ s\end{array}\right) \frac{d^{i-s}}{d x^{i-s}} \circ a_{i, j} \otimes \frac{d^{j+s}}{d x^{j+s}}$. Since $C_{1}$ is linear, formulas (13) are thus proved.

DEFINITION 3.3. We call the modules $\mathcal{D}_{-1-\mu, \lambda_{2},-1-\lambda_{1}}^{k}, \mathcal{D}_{\lambda_{1},-1-\mu,-1-\lambda_{2}}^{k}, \mathcal{D}_{-1-\mu, \lambda_{1}}^{k}$, $-1-\lambda_{2}, \mathcal{D}_{\lambda_{2},-1-\mu,-1-\lambda_{1}}^{k}, \mathcal{D}_{\lambda_{2}, \lambda_{1}, \mu}^{k}$ the adjoint modules of $\mathcal{D}_{\lambda_{1}, \lambda_{2}, \mu}^{k}$.

Especially, $\mathcal{D}_{-1-\mu, \lambda_{2},-1-\lambda_{1}}^{k}$ (respectively $\mathcal{D}_{\lambda_{1},-1-\mu,-1-\lambda_{2}}^{k}$ ) is called the left-adjoint (respectively the right-adjoint) module of $\mathcal{D}_{\lambda_{1}, \lambda_{2}, \mu}^{k}$.

The module $\mathcal{D}_{\lambda_{2}, \lambda_{1}, \mu}^{k}$ is called the symmetric module of $\mathcal{D}_{\lambda_{1}, \lambda_{2}, \mu}^{k}$.

A module of the form $\mathcal{D}_{\lambda, \lambda,-1-\lambda}^{k}, \lambda \in \mathbb{R}$ will be said a self-adjoint module.

A module will be said singular if it is not isomorphic to any module other than itself and previously mentioned modules.

3.3. Explicit formulas for the action of $\operatorname{Vect}(\mathbb{R})$ on $\mathcal{D}_{\lambda_{1}, \lambda_{2}, \mu}^{k}$. Let $X=X(x) \frac{d}{d x} \in$ $\operatorname{Vect}(\mathbb{R})$ and $A=\sum_{\ell=0}^{k} \sum_{i+j=\ell} a_{i, j} \frac{d^{i}}{d x^{i}} \otimes \frac{d^{j}}{d x^{j}} \in \mathcal{D}_{\lambda_{1}, \lambda_{2}, \mu}^{k}$. The following result gives the expression of $L_{X}^{\lambda_{1}, \lambda_{2}, \mu}(A)$.

PROPOSITION 3.4. The action of the vector field $X$ on the operator $A$ is given by

$$
L_{X}^{\lambda_{1}, \lambda_{2}, \mu}(A)=\sum_{\ell=0}^{k} \sum_{i+j=\ell} a_{i, j}^{X} \frac{d^{i}}{d x^{i}} \otimes \frac{d^{j}}{d x^{j}}
$$


where:

(18)

$$
\begin{aligned}
& a_{0,0}^{X}=L_{X}^{\delta}\left(a_{0,0}\right)+\sum_{s=1}^{k}\left(a_{s, 0} \lambda_{1}+a_{0, s} \lambda_{2}\right) X^{(s+1)} \\
& a_{p, 0}^{X}=L_{X}^{\delta+p}\left(a_{p, 0}\right)+\sum_{s=1}^{k-p}\left(a_{p+s, 0}\left(-\left(\begin{array}{c}
s+p \\
s+1
\end{array}\right)+\lambda_{1}\left(\begin{array}{c}
s+p \\
s
\end{array}\right)\right)+a_{p, s} \lambda_{2}\right) X^{(s+1)} ; 1 \leq p \leq k-1 \\
& a_{0, p}^{X}=L_{X}^{\delta+p}\left(a_{0, p}\right)+\sum_{s=1}^{k-p}\left(a_{0, p+s}\left(-\left(\begin{array}{c}
s+p \\
s+1
\end{array}\right)+\lambda_{2}\left(\begin{array}{c}
s+p \\
s
\end{array}\right)\right)+a_{s, p} \lambda_{1}\right) X^{(s+1)} ; 1 \leq p \leq k-1 \\
& a_{i, j}^{X}=L_{X}^{\delta+p}\left(a_{i j}\right)+\sum_{s=1}^{k-p}\left(a_{s+i, j}\left(-\left(\begin{array}{c}
s+i \\
s+1
\end{array}\right)+\lambda_{1}\left(\begin{array}{c}
s+i \\
s+1
\end{array}\right)\right)+a_{i, s+j}\left(-\left(\begin{array}{c}
s+j \\
s+1
\end{array}\right)+\lambda_{2}\left(\begin{array}{c}
s+j \\
s
\end{array}\right)\right)\right) X^{(s+1)} ; \\
& 1 \leq p=i+j \leq k-1 \\
& a_{i, j}^{X}=L_{X}^{\delta+k}\left(a_{i j}\right) ; i+j=k .
\end{aligned}
$$

Proof. Let $\Phi_{1}=\varphi_{1}(x)(d x)^{-\lambda_{1}} \in \mathcal{F}_{\lambda_{1}}, \Phi_{2}=\varphi_{2}(x)(d x)^{-\lambda_{2}} \in \mathcal{F}_{\lambda_{2}}$. Upon using (2) and (8) we get

$$
\begin{aligned}
L_{X}^{\lambda_{1}, \lambda_{2}, \mu}(A)\left(\Phi_{1}, \Phi_{2}\right)= & L_{X}^{\mu} \circ A\left(\Phi_{1}, \Phi_{2}\right)-A\left(L_{X}^{\lambda_{1}}\left(\Phi_{1}\right), \Phi_{2}\right)-A\left(\Phi_{1}, L_{X}^{\lambda_{2}}\left(\Phi_{2}\right)\right) \\
= & (d x)^{-\mu}\left[\sum_{\ell=0}^{k} \sum_{i+j=\ell}\left(a_{i, j} \varphi_{1}^{(i)} \varphi_{2}^{(j)}\right)^{\prime} X-\mu \varphi_{1}^{(i)} \varphi_{2}^{(j)} X^{\prime}\right. \\
& \left.-a_{i, j}\left(\varphi_{1}^{\prime} X-\lambda_{1} \varphi_{1} X^{\prime}\right)^{(i)} \varphi_{2}^{(j)}-a_{i, j} \varphi_{1}^{(i)}\left(\varphi_{2}^{\prime} X-\lambda_{2} \varphi_{2} X^{\prime}\right)^{(j)}\right]
\end{aligned}
$$

On the other hand, from (17), we have

$$
L_{X}^{\lambda_{1}, \lambda_{2}, \mu}(A)\left(\Phi_{1}, \Phi_{2}\right)=(d x)^{-\mu} \sum_{\ell=0}^{k} \sum_{i+j=\ell} a_{i, j}^{X} \varphi_{1}^{(i)} \varphi_{2}^{(j)} .
$$

Then, formulas (18) are easily obtained by identification.

3.4. Space of symbols of bilinear differential operators. Consider the graded $\operatorname{Vect}(\mathbb{R})$-module $\operatorname{gr}\left(D_{\lambda_{1}, \lambda_{2}, \mu}\right)$ associated with the filtration

$$
\mathcal{D}_{\lambda_{1}, \lambda_{2}, \mu}^{0} \subset \mathcal{D}_{\lambda_{1}, \lambda_{2}, \mu}^{1} \subset \cdots \subset \mathcal{D}_{\lambda_{1}, \lambda_{2}, \mu}^{k-1} \subset \mathcal{D}_{\lambda_{1}, \lambda_{2}, \mu}^{k} \subset \cdots
$$

i.e., the direct sum

$$
\operatorname{gr}\left(\mathcal{D}_{\lambda_{1}, \lambda_{2}, \mu}\right)=\bigoplus_{\ell=0}^{\infty} \mathcal{D}_{\lambda_{1}, \lambda_{2}, \mu}^{\ell} / \mathcal{D}_{\lambda_{1}, \lambda_{2}, \mu}^{\ell-1}
$$

with the convention that $\mathcal{D}_{\lambda_{1}, \lambda_{2}, \mu}^{-1}=\{0\}$. We call this $\operatorname{Vect}(\mathbb{R})$-module the space of symbols of bilinear differential operators and denote it $\mathcal{S}_{\lambda_{1}, \lambda_{2}, \mu}$. The space of $k$-th order symbols is

$$
\mathcal{S}_{\lambda_{1}, \lambda_{2}, \mu}^{k}:=\bigoplus_{\ell=0}^{k} \mathcal{D}_{\lambda_{1}, \lambda_{2}, \mu}^{\ell} / \mathcal{D}_{\lambda_{1}, \lambda_{2}, \mu}^{\ell-1}
$$


Proposition 3.5. The Vect $(\mathbb{R})$-module $\mathcal{S}_{\lambda_{1}, \lambda_{2}, \mu}$ depends only on the shift, $\delta=\mu-$ $\lambda_{1}-\lambda_{2}$, of the weights and not on $\mu, \lambda_{1}$ and $\lambda_{2}$ independently. Moreover, for every $k \in \mathbb{N}$, we have

$$
\mathcal{S}_{\lambda_{1}, \lambda_{2}, \mu}^{k}=\mathcal{S}_{\delta}^{k} \cong \bigoplus_{\ell=0}^{k} \bigoplus_{i+j=\ell} \mathcal{F}_{\frac{\delta}{2}+i} \otimes \mathcal{F}_{\frac{\delta}{2}+j} .
$$

PROOF. We consider the projection called the principal symbol map

$$
\sigma_{\mathrm{pr}}: \mathcal{D}_{\lambda_{1}, \lambda_{2}, \mu}^{k} \rightarrow \bigoplus_{i+j=k} \mathcal{F}_{\frac{\delta}{2}+i} \otimes \mathcal{F}_{\frac{\delta}{2}+j}
$$

defined for $A=\sum_{\ell=0}^{k} \sum_{i+j=\ell} a_{i, j}(x) \frac{d^{i}}{d x^{i}} \otimes \frac{d^{j}}{d x^{j}} \in \mathcal{D}_{\lambda_{1}, \lambda_{2}, \mu}^{k}$ by:

$$
\sigma_{\mathrm{pr}}(A):=\sum_{i+j=k} a_{i, j}(x) d x^{\frac{\delta}{2}+i} \otimes d x^{\frac{\delta}{2}+j} .
$$

One easily checks that $\sigma_{\mathrm{pr}}$ is a $\operatorname{Vect}(\mathbb{R})$-equivariant map.

REMARK 3.6. Let $(x, \xi)$ a standard coordinate of $T^{*} \mathbb{R}$, we can readily see that the space of symbols $\mathcal{S}_{\delta}$, being treated as a Vect $(\mathbb{R})$-module, is isomorphic to $\mathcal{F}_{\delta} \otimes C^{\infty}(\mathbb{R}) \otimes$ $\mathbb{R}[\xi]^{\otimes 2}$. It will be convenient, in the sequel, to write an element of $\mathcal{S}_{\delta}^{k}$ in the form

$$
P(x, \xi)=\xi^{\delta} \sum_{\ell=0}^{k} \sum_{i+j=\ell} \bar{a}_{i, j}(x) \xi^{i} \otimes \xi^{j} .
$$

4. Projectively equivariant symbol and quantization maps. A map $\sigma: \mathcal{D}_{\lambda_{1}, \lambda_{2}, \mu} \rightarrow$ $\mathcal{S}_{\delta}$ is called a symbol map if it is bijective and for every $k \in \mathbb{N}$ the following diagram is commutative:

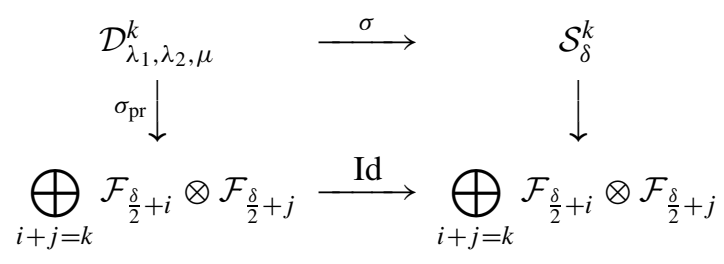

where the right arrow is the projection. In other words, the highest-order term of $\sigma$ coincides with the principal symbol map defined in (23). The inverse map, $Q=\sigma^{-1}$, is called the quantization map. The following statement is the main result of this paper it shows that for generic values of $\delta$ the $\mathrm{sl}(2, \mathbb{R})$-module $\mathcal{D}_{\lambda_{1}, \lambda_{2}, \mu}^{k}$ is isomorphic to $\mathcal{S}_{\delta}^{k}$. The $\mathrm{sl}(2, \mathbb{R})$-equivariant map is called a projectively equivariant symbol mapping.

The main theorem of the paper is the following

THEOREM 4.1. If $\delta=\mu-\lambda_{1}-\lambda_{2} \neq-1,-3 / 2,-2,-5 / 2, \ldots,-k$, then $\mathcal{D}_{\lambda_{1}, \lambda_{2}, \mu}^{k}$ is isomorphic to $\mathcal{S}_{\delta}^{k}$ as an $\operatorname{sl}(2, \mathbb{R})$-module. The isomorphism is given by the unique (up to 
normalization) following $\operatorname{sl}(2, \mathbb{R})$-equivariant symbol map

$$
\sigma_{\lambda_{1}, \lambda_{2}, \mu}(A)=\xi^{\delta} \sum_{p=0}^{k} \sum_{i+j=p}\left(\sum_{\ell=p}^{k} \sum_{\substack{s+t=\ell \\
s \geq i, t \geq j}} \frac{\left(\begin{array}{c}
s \\
i
\end{array}\right)\left(\begin{array}{c}
t \\
j
\end{array}\right)\left(\begin{array}{c}
2 \lambda_{1}-i \\
2 \lambda_{1}-s
\end{array}\right)\left(\begin{array}{c}
2 \lambda_{2}-j \\
2 \lambda_{2}-t
\end{array}\right)}{\left(\begin{array}{c}
\ell-p \\
s-i
\end{array}\right)\left(\begin{array}{c}
2 \delta+\ell+p+1 \\
2 \delta+2 p+1
\end{array}\right)} a_{s, t}^{(\ell-p)}\right) \xi^{i} \otimes \xi^{j}
$$

where $A=\sum_{p=0}^{k} \sum_{i+j=p} a_{i, j} \frac{d^{i}}{d x^{i}} \otimes \frac{d^{j}}{d x^{j}} \in \mathcal{D}_{\lambda_{1}, \lambda_{2}, \mu}^{k}$. Note that the binomial coefficients in (27) are defined by $\left(\begin{array}{l}v \\ q\end{array}\right)=\frac{v(v-1) \cdots(v-q+1)}{q !}$. This expression makes sense for arbitrary $v \in \mathbb{C}$.

Proof. Let us denote by

$$
\alpha_{i, j}^{s, t}=\left\{\begin{array}{l}
\frac{\left(\begin{array}{l}
s \\
i
\end{array}\right)\left(\begin{array}{c}
t \\
j
\end{array}\right)\left(\begin{array}{c}
2 \lambda_{1}-i \\
2 \lambda_{1}-s
\end{array}\right)\left(\begin{array}{c}
2 \lambda_{2}-j \\
2 \lambda_{2}-t
\end{array}\right)}{\left(\begin{array}{c}
\ell-p \\
s-i
\end{array}\right)\left(\begin{array}{c}
2 \delta+\ell+p+1 \\
2 \delta+2 p+1
\end{array}\right)} \text { if } s \geq i, t \geq j, \\
\alpha_{i, j}^{s, t}=0 \text { otherwhise }
\end{array}\right.
$$

where $\ell=s+t, p=i+j$ and

$$
\sigma_{\lambda_{1}, \lambda_{2}, \mu}\left(\left(L_{X}^{\lambda_{1}, \lambda_{2}, \mu}(A)\right)=\xi^{\delta} \sum_{p=0}^{k} \sum_{i+j=p} \bar{a}_{i, j}^{X}(x) \xi^{i} \otimes \xi^{j},\right.
$$

where $X \in \operatorname{Vect}(\mathbb{R})$. Then, we readily see that

$$
\bar{a}_{i, j}^{X}(x)=\sum_{\ell=p}^{k} \sum_{s+t=\ell} \alpha_{i, j}^{s, t}\left(a_{s, t}^{X}\right)^{(\ell-p)} ; p=i+j .
$$

Thanks to Proposition 3.4, forall $0 \leq p=i+j \leq k$, we get (30)

$$
\begin{aligned}
& \bar{a}_{i, j}^{X}=\sum_{s+t=p} \alpha_{i, j}^{s, t}\left(L_{X}^{\delta+p}\left(a_{s, t}\right)+\sum_{m=1}^{k-p}\left[a_{m+s, t}\left(-\left(\begin{array}{c}
m+s \\
m+1
\end{array}\right)+\lambda_{1}\left(\begin{array}{c}
m+s \\
m
\end{array}\right)\right)+\right.\right. \\
& \left.\left.a_{s, t+m}\left(-\left(\begin{array}{c}
m+t \\
m+1
\end{array}\right)+\lambda_{2}\left(\begin{array}{c}
m+t \\
m
\end{array}\right)\right)\right] X^{(m+1)}\right)+ \\
& \sum_{\ell=p+1}^{k-1} \sum_{\substack{s+t=\ell \\
s \neq 0, t \neq 0}} \alpha_{i, j}^{s, t}\left(L_{X}^{\delta+\ell}\left(a_{s, t}\right)+\sum_{m=1}^{k-\ell}\left[a_{m+s, t}\left(-\left(\begin{array}{c}
m+s \\
m+1
\end{array}\right)+\lambda_{1}\left(\begin{array}{c}
m+s \\
m
\end{array}\right)\right)+\right.\right. \\
& \left.\left.a_{s, t+m}\left(-\left(\begin{array}{c}
m+t \\
m+1
\end{array}\right)+\lambda_{2}\left(\begin{array}{c}
m+t \\
m
\end{array}\right)\right)\right] X^{(m+1)}\right)^{(\ell-p)}+ \\
& \sum_{\substack{k-1 \\
k-s}} \alpha_{i, j}^{s, 0}\left(L_{X}^{\delta+s}\left(a_{s, 0}\right)+\sum_{m=1}^{k+1}\left[a_{m+s, 0}\left(-\left(\begin{array}{c}
m+s \\
m+1
\end{array}\right)+\lambda_{1}\left(\begin{array}{c}
m+s \\
m
\end{array}\right)\right)+a_{s, m} \lambda_{2}\right] X^{(m+1)}\right)^{(s-p)}+ \\
& \sum_{k-1} \alpha_{i, j}^{0, t}\left(L_{X}^{\delta+t}\left(a_{0, t}\right)+\sum_{m=1}^{k-t}\left[a_{0, m+t}\left(-\left(\begin{array}{c}
m+t \\
m+1
\end{array}\right)+\lambda_{2}\left(\begin{array}{c}
m+t \\
m
\end{array}\right)\right)+a_{m, t} \lambda_{1}\right] X^{(m+1)}\right)^{(t-p)}+ \\
& \sum_{t=p+1} \alpha_{i, j}^{s, t}\left(L_{X}^{(\delta+k)}\left(a_{s, t}\right)\right)^{(k-p)} . \\
& s+t=k
\end{aligned}
$$


Thus

(31)

$$
\begin{aligned}
& \bar{a}_{i, j}^{X}-L_{X}^{(\delta+p)}\left(\bar{a}_{i, j}\right)=\left[\sum_{s+t=p+1}-\alpha_{i, j}^{s, t} a_{s, t}(\delta+p+1)+\right. \\
& \sum_{\ell=p+2 s+t=\ell}^{k} \sum_{i, j}^{s, t}\left(\left(\begin{array}{c}
\ell-p \\
2
\end{array}\right)-\left(\begin{array}{c}
\ell-p \\
1
\end{array}\right)(\delta+\ell)\right) a_{s, t}^{(\ell-p-1)}+ \\
& \sum_{\ell=p s+t=\ell}^{k-1} \sum_{i, j}^{s, t}\left(\left(-\left(\begin{array}{c}
s+1 \\
2
\end{array}\right)+\lambda_{1}\left(\begin{array}{c}
s+1 \\
1
\end{array}\right)\right) a_{s+1, t}^{(\ell-p)}+\left(-\left(\begin{array}{c}
t+1 \\
2
\end{array}\right)+\lambda_{2}\left(\begin{array}{c}
t+1 \\
1
\end{array}\right)\right) a_{s, t+1}^{(\ell-p)}\right)+ \\
& \sum_{s=p+1}^{k-1} \alpha_{i, j}^{s, 0}\left(\left(-\left(\begin{array}{c}
s+1 \\
2
\end{array}\right)+\lambda_{1}\left(\begin{array}{c}
s+1 \\
1
\end{array}\right)\right) a_{s+1,0}^{(s-p)}+\lambda_{2} a_{s, 1}^{(s-p)}\right)+ \\
& \left.\sum_{t=p+1}^{k-1} \alpha_{i, j}^{0, t}\left(\left(-\left(\begin{array}{c}
t+1 \\
2
\end{array}\right)+\lambda_{2}\left(\begin{array}{c}
t+1 \\
1
\end{array}\right)\right) a_{0, t+1}^{(t-p)}+\lambda_{1} a_{1, t}^{(t-p)}\right)\right] X^{\prime \prime}+\text { terms in } X^{(n)} ;(n \geq 3) .
\end{aligned}
$$

Since $\alpha_{i, j}^{s, t}$ satisfies the equation

$$
(\ell-p)(2 \delta+\ell+p+1) \alpha_{i, j}^{s, t}-s\left(2 \lambda_{1}-s+1\right) \alpha_{i, j}^{s-1, t}-t\left(2 \lambda_{2}-t+1\right) \alpha_{i, j}^{s, t-1}=0,
$$

the term in $X^{\prime \prime}$ vanishes and the map $\sigma_{\lambda_{1}, \lambda_{2}, \mu}$ is clearly $\mathrm{sl}(2, \mathbb{R})$-equivariant.

Let $\chi: \mathcal{D}_{\lambda_{1}, \lambda_{2}, \mu} \rightarrow \mathcal{S}_{\delta}$ be an $\operatorname{sl}(2, \mathbb{R})$-equivariant symbol mapping defined by

$$
\bar{a}_{i, j}=\sum_{\ell, r=1}^{k} \sum_{s+t=\ell} \chi_{i, j}^{s, t, r} a_{s, t}^{(r)},
$$

where the $\chi_{i, j}^{s, t, r}$ are generally in $C^{\infty}(\mathbb{R})$. The $\mathrm{sl}(2, \mathbb{R})$-equivariance of $\chi$ means that

$$
\bar{a}_{i, j}^{X}=L_{X}^{\delta+p}\left(\bar{a}_{i, j}\right), \forall 0 \leq p=i+j \leq k, \forall X \in \operatorname{sl}(2, \mathbb{R}) .
$$

The equivariance of $\chi$ with respect to the vector fields $X_{1}=\frac{d}{d x}$ quickly leads to $\frac{d \chi_{i, j}^{s, t, r}}{d x}=0$. Indeed, we have

$$
\bar{a}_{i, j}^{X_{1}}=\sum_{\ell, r=1}^{k} \sum_{s+t=\ell} \chi_{i, j}^{s, t, r}\left(a_{s, t}^{X_{1}}\right)^{(r)}=\sum_{\ell, r=1}^{k} \sum_{s+t=\ell} \chi_{i, j}^{s, t, r}\left(a_{s, t}^{\prime}\right)^{(r)}
$$

and

$$
L_{X_{1}}^{\delta+p}\left(\bar{a}_{i, j}\right)=\left(\bar{a}_{i, j}\right)^{\prime}=\sum_{\ell, r=1}^{k} \sum_{s+t=\ell}\left(\chi_{i, j}^{s, t, r} a_{s, t}^{(r)}\right)^{\prime} .
$$

Thus, the equivariance condition (34) provides the conclusion. By a similar reasoning, thanks to the following equation

$$
L_{X_{x}}^{\delta+p}\left(\bar{a}_{i, j}\right)=x\left(\bar{a}_{i, j}\right)^{\prime}-(p+\delta) \bar{a}_{i, j}
$$


where $X_{x}=x \frac{d}{d x}$, the equivariance of $\chi$ with respect to the vector fields $X_{x}$ leads to the equation

$$
\sum_{\ell, r=1}^{k} \sum_{s+t=\ell} \chi_{i, j}^{s, t, r}\left(x a_{s, t}^{(r+1)}-(\delta+\ell-r) a_{s, t}^{(r)}\right)=\sum_{\ell, r=1}^{k} \sum_{s+t=\ell} \chi_{i, j}^{s, t, r}\left(x a_{s, t}^{(r+1)}-(\delta+p) a_{s, t}^{(r)}\right)
$$

which means that $\bar{a}_{i, j}=\sum_{\ell=1}^{k} \sum_{s+t=\ell} \chi_{i, j}^{s, t} a_{s, t}^{(\ell-p)}$. The map $\chi$ is bijective if and only if, forall $p \in\{0,1, \ldots, k\}$, the matrix $\left(\chi_{i, p-i}^{j, p-j}\right)_{\substack{0 \leq i \leq p \\ 0 \leq j \leq p}}$ is invertible.

Finally, if we impose the equivariance with respect to the vector fields $X_{x^{2}}=x^{2} \frac{d}{d x}$, as the previous cases and given that

$$
L_{X_{x^{2}}}^{\delta+p}\left(\bar{a}_{i, j}\right)=x^{2}\left(\bar{a}_{i, j}\right)^{\prime}-2(p+\delta) x \bar{a}_{i, j},
$$

by a direct computation, we get

$$
\chi_{i, j}^{s, t}=\frac{s\left(2 \lambda_{1}-s+1\right) \chi_{i, j}^{s-1, t}+t\left(2 \lambda_{2}-t+1\right) \chi_{i, j}^{s, t-1}}{(\ell-p)(2 \delta+\ell+p+1)} ; s+t \geq p, s \geq 1, t \geq 1
$$

and

$$
\chi_{i, j}^{s, 0}=\frac{\left(\begin{array}{c}
s \\
p
\end{array}\right)\left(\begin{array}{c}
2 \lambda_{1}-p \\
2 \lambda_{1}-s
\end{array}\right)}{\left(\begin{array}{c}
2 \delta+s+p+1 \\
2 \delta+2 p+1
\end{array}\right)} \chi_{i, j}^{p, 0} ; \chi_{i, j}^{0, t}=\frac{\left(\begin{array}{c}
t \\
p
\end{array}\right)\left(\begin{array}{c}
2 \lambda_{2}-p \\
2 \lambda_{2}-t
\end{array}\right)}{\left(\begin{array}{c}
2 \delta+t+p+1 \\
2 \delta+2 p+1
\end{array}\right)} \chi_{i, j}^{0, p} ; p+1 \leq s, t \leq k
$$

If we set $\left(\chi_{i, p-i}^{j, p-j}\right)_{\substack{0 \leq i \leq p \\ 0 \leq j \leq p}}=I_{p+1}$ for all $p \in\{0,1, \ldots, k\}$, we obtain $\chi_{i, j}^{s, t}=\alpha_{i, j}^{s, t} ; \forall i, j, s, t$. The uniqness, up to normalization, of $\sigma_{\lambda_{1}, \lambda_{2}, \mu}$ is thus proved.

Let us now give the explicit formula for the quantization map.

PROPOSITION 4.2. The map $Q_{\lambda_{1}, \lambda_{2}, \mu}=\sigma_{\lambda_{1}, \lambda_{2}, \mu}^{-1}$ associates to a polynomial $P(x, \xi)=\xi^{\delta} \sum_{\ell=0}^{k} \sum_{i+j=\ell} b_{i, j}(x) \xi^{i} \otimes \xi^{j} \in \mathcal{S}_{\delta}^{k}$ the differential operator $Q_{\lambda_{1}, \lambda_{2}, \mu}(P)=$ $\sum_{\ell=0}^{k} \sum_{i+j=\ell} \tilde{b}_{i, j} \frac{d^{i}}{d x^{i}} \otimes \frac{d^{j}}{d x^{j}} \in \mathcal{D}_{\lambda_{2}, \lambda_{1}, \mu}^{k}$ such that $\tilde{b}_{i, j}=\sum_{\ell=p}^{k} \sum_{s+t=\ell} \beta_{i, j}^{s, t} b_{s, t}^{(\ell-p)}$, where

$$
\left\{\begin{array}{l}
\beta_{i, j}^{s, t}=\sum_{m=1}^{\ell-p}(-1)^{m} \sum_{p \leq i_{1}+j_{1}<i_{2}+j_{2}<\cdots<i_{m-1}+j_{m-1} \leq \ell} \\
\beta_{i, j}^{s, t}=\alpha_{i, j}^{s, t} \text { if } s+t=i+j .
\end{array} \alpha_{i, j}^{i_{1}, j_{1}} \alpha_{i_{1}, j_{1}}^{i_{2}, j_{2}} \cdots \alpha_{i_{m-1}, j_{m-1}}^{s, t} \text { if } \ell=s+t>p=i+j\right.
$$

5. Intertwining operators. The uniqueness, up to normalization, of the map $\sigma_{\lambda_{1}, \lambda_{2}, \mu}$ implies that in terms of the $\mathrm{sl}(2, \mathbb{R})$-equivariant symbol, every isomorphisms of modules of bilinear differential operators has a 'diagonal' form in the following sense

THEOREM 5.1. Let $\delta=\mu-\lambda_{1}-\lambda_{2} \neq-1,-3 / 2,-2,-5 / 2, \ldots,-k$ and $T$ : $\mathcal{D}_{\lambda_{1}, \lambda_{2}, \mu}^{k} \rightarrow \mathcal{D}_{\lambda_{1}, \lambda_{2}, \mu}^{k}$ be a linear map. 
For a given $A=\sum_{p=0}^{k} \sum_{i=0}^{p} a_{i, p-i} \frac{d^{i}}{d x^{i}} \otimes \frac{d^{p-i}}{d x^{p-i}}$ in $\mathcal{D}_{\lambda_{1}, \lambda_{2}, \mu}^{k}$, we denote by

(43) $\sigma_{\lambda_{1}, \lambda_{2}, \mu}^{p}(A)=\xi^{\delta} \sum_{i=0}^{p} \bar{a}_{i, p-i} \xi^{i} \otimes \xi^{p-i}$ and $\sigma_{\lambda_{1}, \lambda_{2}, \mu}^{p}(T(A))=\xi^{\delta} \sum_{i=0}^{p} \bar{a}_{i, p-i}^{T} \xi^{i} \otimes \xi^{p-i}$

the homogeneous components of order $p$ of $\sigma_{\lambda_{1}, \lambda_{2}, \mu}(A)$ and $\sigma_{\lambda_{1}, \lambda_{2}, \mu}(T(A))$ respectively. Then, $T$ is sl(2)-equivariant if and only if, for all $p \in\{0,1, \ldots, k\}$ the symbols $\sigma_{\lambda_{1}, \lambda_{2}, \mu}^{p}(A)$ and $\sigma_{\lambda_{1}, \lambda_{2}, \mu}^{p}(T(A))$ are 'proportional', that is there exits an invertible matrix $\Upsilon_{p}=\left(\varepsilon_{i, p-i}^{j, p-j}\right)_{\substack{0 \leq i \leq p \\ 0 \leq j \leq p}} \in G L_{p+1}(\mathbb{R})$ such that

$$
\left(\bar{a}_{0, p}^{T}, \bar{a}_{1, p-1}^{T}, \ldots, \bar{a}_{p, 0}^{T}\right)^{t}=\Upsilon_{p}\left(\bar{a}_{0, p}, \bar{a}_{1, p-1}, \ldots, \bar{a}_{p, 0}\right)^{t}
$$

where the notation $u^{t}$ means the transpose of the vector $u \in \mathbb{R}^{n}$. Equivalently,

$$
\bar{a}_{i, p-i}^{T}=\sum_{j=0}^{p} \varepsilon_{i, p-i}^{j, p-j} \bar{a}_{j, p-j} ; \forall i \in\{0,1, \ldots, p\} .
$$

In other words, the map $\sigma_{\lambda_{1}, \lambda_{2}, \mu} \circ T \circ \sigma_{\lambda_{1}, \lambda_{2}, \mu}^{-1}$ on $\mathcal{S}_{\delta}^{0} \bigoplus \mathcal{S}_{\delta}^{1} \cdots \bigoplus \mathcal{S}_{\delta}^{k}$ multiplies each term of the direct sum by an invertible matrix.

6. Example: second order bilinear differential operators. In this section we plan the case of the modules $\mathcal{D}_{\lambda_{1}, \lambda_{2}, \mu}^{2}$ of second order bilinear differential operators with the condition $\delta=\mu-\lambda_{1}-\lambda_{2} \neq\{-1,-3 / 2,-2\}$. Let $A=\sum_{\ell=0}^{2} \sum_{i+j=\ell} a_{i, j} \frac{d^{i}}{d x^{i}} \otimes \frac{d^{j}}{d x^{j}} \in \mathcal{D}_{\lambda_{1}, \lambda_{2}, \mu}^{2}$ and $P=\xi^{\delta} \sum_{\ell=0}^{2} \sum_{i+j=\ell} b_{i, j} \xi^{i} \otimes \xi^{j} \in \mathcal{S}_{\delta}^{2}$. By Proposition 3.4, the action of $\operatorname{Vect}(\mathbb{R})$ on $\mathcal{D}_{\lambda_{2}, \lambda_{1}, \mu}^{2}$ is given by

$$
\left\{\begin{array}{l}
a_{0,0}^{X}=L_{X}^{\delta}\left(a_{0,0}\right)+\left(a_{1,0} \lambda_{1}+a_{0,1} \lambda_{2}\right) X^{\prime \prime}+\left(a_{2,0} \lambda_{1}+a_{0,2} \lambda_{2}\right) X^{\prime \prime \prime} \\
a_{0,1}^{X}=L_{X}^{\delta+1}\left(a_{0,1}\right)+\left(a_{0,2}\left(2 \lambda_{2}-1\right)+a_{1,1} \lambda_{1}\right) X^{\prime \prime} \\
a_{1,0}^{X}=L_{X}^{\delta+1}\left(a_{1,0}\right)+\left(a_{2,0}\left(2 \lambda_{1}-1\right)+a_{1,1} \lambda_{2}\right) X^{\prime \prime} \\
a_{0,2}^{X}=L_{X}^{\delta+2}\left(a_{0,2}\right) \\
a_{1,1}^{X}=L_{X}^{\delta+2}\left(a_{1,1}\right) \\
a_{2,0}^{X}=L_{X}^{\delta+2}\left(a_{2,0}\right) .
\end{array}\right.
$$

If we set

$$
\sigma_{\lambda_{1}, \lambda_{2}, \mu}(A)=\xi^{\delta} \sum_{p=0}^{2} \sum_{i+j=p} \bar{a}_{i, j} \xi^{i} \otimes \xi^{j}
$$

and

$$
Q_{\lambda_{1}, \lambda_{2}, \mu}(P)=\sum_{\ell=0}^{2} \sum_{i+j=\ell} \tilde{b}_{i, j} \frac{d^{i}}{d x^{i}} \otimes \frac{d^{j}}{d x^{j}}
$$


from (27) and (42), the sl(2)-equivariant symbol and quantization maps are respectively given by the following expressions

(49)

$$
\left\{\begin{array}{l}
\bar{a}_{0,0}=a_{0,0}+\frac{\lambda_{2}}{\delta+1} a_{0,1}^{\prime}+\frac{\lambda_{1}}{\delta+1} a_{1,0}^{\prime}+\frac{\lambda_{2}\left(2 \lambda_{2}-1\right)}{(\delta+1)(2 \delta+3)} a_{0,2}^{\prime \prime}+\frac{2 \lambda_{1} \lambda_{2}}{(\delta+1)(2 \delta+3)} a_{1,1}^{\prime \prime}+\frac{\lambda_{1}\left(2 \lambda_{1}-1\right)}{(\delta+1)(2 \delta+3)} a_{2,0}^{\prime \prime} \\
\bar{a}_{0,1}=a_{0,1}+\frac{2 \lambda_{2}-1}{\delta+2} a_{0,2}^{\prime}+\frac{\lambda_{1}}{\delta+2} a_{1,1}^{\prime} \\
\bar{a}_{1,0}=a_{1,0}+\frac{2 \lambda_{1}-1}{\delta+2} a_{2,0}^{\prime}+\frac{\lambda_{2}}{\delta+2} a_{1,1}^{\prime} \\
\bar{a}_{0,2}=a_{0,2} \\
\bar{a}_{1,1}=a_{1,1} \\
\bar{a}_{2,0}=a_{2,0}
\end{array}\right.
$$

and

(50)

$$
\left\{\begin{array}{l}
\tilde{b}_{0,0}=b_{0,0}-\frac{\lambda_{2}}{\delta+1} b_{0,1}^{\prime}-\frac{\lambda_{1}}{\delta+1} b_{1,0}^{\prime}+\frac{\lambda_{2}\left(2 \lambda_{2}-1\right)}{(\delta+2)(2 \delta+3)} b_{0,2}^{\prime \prime}+\frac{2 \lambda_{1} \lambda_{2}}{(\delta+2)(2 \delta+3)} b_{1,1}^{\prime \prime}+\frac{\lambda_{1}\left(2 \lambda_{1}-1\right)}{(\delta+2)(2 \delta+3)} b_{2,0}^{\prime \prime} \\
\tilde{b}_{0,1}=b_{0,1}-\frac{2 \lambda_{2}-1}{\delta+2} a_{0,2}^{\prime}-\frac{\lambda_{1}}{\delta+2} a_{1,1}^{\prime} \\
\tilde{b}_{1,0}=b_{1,0}-\frac{2 \lambda_{1}-1}{\delta+2} b_{2,0}^{\prime}-\frac{\lambda_{2}}{\delta+2} b_{1,1}^{\prime} \\
\tilde{b}_{0,2}=b_{0,2} \\
\tilde{b}_{1,1}=b_{1,1} \\
\tilde{b}_{2,0}=b_{2,0}
\end{array}\right.
$$

Let us now give a complete classification of the modules $\mathcal{D}_{\lambda_{1}, \lambda_{2}, \mu}^{2}\left(\delta=\mu-\lambda_{1}-\lambda_{2} \neq\right.$ $\{-1,-3 / 2,-2\})$

THEOREM 6.1. (i) All the Vect $(\mathbb{R})$-modules $\mathcal{D}_{\lambda_{1}, \lambda_{2}, \mu}^{2}$ with $\left(\lambda_{1} \neq 0\right.$ and $\left.\lambda_{2} \neq 0\right),\left(\lambda_{1} \neq 0\right.$ and $\mu \neq-1),\left(\lambda_{2} \neq 0\right.$ and $\left.\mu \neq-1\right)$ are isomorphic.

(ii) The modules of the form

$$
\mathcal{D}_{\lambda, 0,-1}^{2} \cong \mathcal{D}_{0, \lambda,-1}^{2} \cong \mathcal{D}_{0,0,-1-\lambda}^{2} ; \lambda \neq 0
$$

are singular.

The module $\mathcal{D}_{0, \lambda,-1}^{2}$ (respectively $\mathcal{D}_{\lambda, 0,-1}^{2}$ ) is a self-left-adjoint (respectively self-rightadjoint) module.

PROOF. Let $T: \mathcal{D}_{\lambda_{1}, \lambda_{2}, \mu}^{2} \rightarrow \mathcal{D}_{\rho_{1}, \rho_{2}, \nu}^{2}$ be an isomorphim of $\operatorname{Vect}(\mathbb{R})$-modules then obviously $\delta=\mu-\lambda_{1}-\lambda_{2}=v-\rho_{1}-\rho_{2}$. Denote by $\widetilde{T}:=\sigma_{\rho_{1}, \rho_{2}, v} \circ T \circ \sigma_{\lambda_{1}, \lambda_{2}, \mu}^{-1}: \mathcal{S}_{\delta}^{2} \rightarrow$ $\mathcal{S}_{\delta}^{2}$. Using Theorem 5.1, $\widetilde{T}$ is 'diagonal', that is there exit $\varepsilon_{0} \in \mathbb{R}^{*}, \Upsilon_{1}=\left(\begin{array}{cc}\varepsilon_{0,1}^{0,1} & \varepsilon_{0,1}^{1,0} \\ \varepsilon_{1,0}^{0,1} & \varepsilon_{1,0}^{1,0}\end{array}\right) \in$ $G L(2, \mathbb{R})$ and

$$
\begin{aligned}
& \Upsilon_{2}=\left(\begin{array}{lll}
\varepsilon_{0,2}^{0,2} & \varepsilon_{0,2}^{1,1} & \varepsilon_{0,2}^{2,0} \\
\varepsilon_{1,2}^{0,2} & \varepsilon_{1,1}^{1,1} & \varepsilon_{1,0}^{2,1} \\
\varepsilon_{2,0}^{0,2} & \varepsilon_{2,0}^{1,1} & \varepsilon_{2,0}^{2,0}
\end{array}\right) \in G L(3, \mathbb{R}) \text { such that } \\
& \bar{a}_{0,0}^{T}=\varepsilon_{0} \bar{a}_{0,0},\left(\bar{a}_{0,1}^{T}, \bar{a}_{1,0}^{T}\right)^{t}=\Upsilon_{1}\left(\bar{a}_{0,1}, \bar{a}_{1,0}\right)^{t} \text { and }\left(\bar{a}_{0,2}^{T}, \bar{a}_{1,1}^{T}, \bar{a}_{2,0}^{T}\right)^{t}=\Upsilon_{2}\left(\bar{a}_{0,2}, \bar{a}_{1,1}, \bar{a}_{2,0}\right)^{t}
\end{aligned}
$$


we set for $a, b, c \in \mathbb{R}$,

$$
\Gamma(a, b, c)=\left(\frac{2 b(c-a+1)}{2(c-a-b)+3}, \frac{2 a b}{2(c-a-b)+3}, \frac{2 a(c-b+1)}{2(c-a-b)+3}\right),
$$

then by a direct computation, the equivariance condition

$$
L_{X}^{\rho_{1}, \rho_{2}, \nu} \circ T=T \circ L_{X}^{\lambda_{1}, \lambda_{2}, \mu},
$$

leads us (in terms of equivariant symbols) to the following matrix equation

$$
\Upsilon_{2} \Gamma\left(\rho_{1}, \rho_{2}, v\right)^{t}=\varepsilon_{0} \Gamma\left(\lambda_{1}, \lambda_{2}, \mu\right)^{t} .
$$

Since $T$ is an isomorphism, two cases arise

i) $\Gamma\left(\rho_{1}, \rho_{2}, v\right) \neq 0$ and $\Gamma\left(\lambda_{1}, \lambda_{2}, \mu\right) \neq 0$, that is $\left(\rho_{1}, \rho_{2}, v\right)$ and $\left(\lambda_{1}, \lambda_{2}, \mu\right)$ are in the set

$$
\left\{(a, b, c) \in \mathbb{R}^{3}:(a \neq 0 \text { and } b \neq 0),(a \neq 0 \text { and } c \neq-1),(b \neq 0 \text { and } c \neq-1)\right\} .
$$

Thus we get a family of $\operatorname{Vect}(\mathbb{R})$-isomorphisms given by the conditions

$$
\varepsilon_{0}=1, \Upsilon_{1} \in G L(2, \mathbb{R}) \text { and } \Upsilon_{2} \Gamma\left(\rho_{1}, \rho_{2}, \nu\right)^{t}=\Gamma\left(\lambda_{1}, \lambda_{2}, \mu\right)^{t}
$$

ii) $\Gamma\left(\rho_{1}, \rho_{2}, v\right)=\Gamma\left(\lambda_{1}, \lambda_{2}, \mu\right)=0$, then

$$
\left(\rho_{1}, \rho_{2}, \nu\right),\left(\lambda_{1}, \lambda_{2}, \mu\right) \in\left\{(\lambda, 0,-1),(0, \lambda,-1),(0,0,-1-\lambda) ; \lambda \in \mathbb{R}^{*}\right\} .
$$

This concludes the proof of the theorem.

\section{REFERENCES}

[1] P. Cohen, Yu. Manin And D. Zagier, Automorphic pseudodifferential operators, Algebraic aspects of integrable systems, Progr. Nonlinear. Differential. Equations. Appl. 26 (1997), 17-47.

[2 ] C. Duval, P. Lecomte And V. Ovsienko, Conformally equivariant quantization: existence and uniqueness, Ann. Inst. Fourier 49 (1999), no. 6, 1999-2029.

[ 3 ] H. Gargoubi, Sur la géométrie de l'espace des opérateurs différentiels linéaires sur $\mathbb{R}$, Bull. Soc. Roy. Sci. Liège 69 (2000), no. 1, 21-47.

[ 4 ] H. Gargoubi And V. Ovsienko, Modules of differential operators on the real line, Funct. Anal. Appl. 35 (2001), no.1, 13-18.

[ 5 ] H. Gargoubi, N. Mallouli and V. Ovsienko, Differential operators on supercircle: Conformally equivariant quantization and symbol calculus, Lett. Math. Phys. 79 (2007), 51-65.

[ 6 ] P. Lecomte And V. Ovsienko, Projectively invariant symbol calculus, Lett. Math. Phys. 49 (1999), no. 3, 173-196.

DEPARTEMENT OF MATHEMATICS

FACULTY OF SCIENCES

BP 11713000 SFAX

TUNISIA

E-mail addresses: taher-bechr@hotmail.fr, jamel_boujelben@hotmail.fr, Khaled_286@yahoo.fr 